\title{
Coupling Optimization and Dynamic Simulation for Preventive-Corrective Control of Voltage Instability
}

\author{
Florin Capitanescu, Thierry Van Cutsem, Fellow, IEEE, and Louis Wehenkel, Member, IEEE
}

\begin{abstract}
This paper proposes an approach coupling security-constrained optimal power flow with time-domain simulation to determine an optimal combination of preventive and corrective controls ensuring a voltage stable transition of the system towards a feasible long-term equilibrium, if any of a set of postulated contingencies occurs. A security-constrained optimal power flow is used to adjust the respective contribution of preventive and corrective actions. Furthermore, information is extracted from (quasi steady-state) time-domain simulations to iteratively adjust the set of coupling constraints used by a corrective security-constrained optimal power flow until its solution is found dynamically secure and viable. Numerical results are provided on a realistic 55-bus test system.
\end{abstract}

Index Terms-Emergency control, optimal power flow, preventive control, time-domain simulation, voltage stability.

\section{INTRODUCTION}

$\mathbf{T}$ HE optimal power flow (OPF) problem [1], [2] is a nonlinear, nonconvex, static, large-scale optimization problem which aims at optimizing control means while satisfying some equality constraints (e.g., power flow equations) and inequality constraints (e.g., system operation limits).

The need to take into account constraints on the performance of the system subjected to contingencies has led to the security-constrained OPF problem [3], which has been formulated under "preventive" [3] and "corrective" [4] modes. The latter, referred to as CSCOPF in the sequel, allows for adjusting controls, other than automatic ones, in post-contingency states. One underlying assumption is that post-contingency constraint violations (e.g., thermal limits, voltage bounds, etc.) can be tolerated for some time without damaging the corresponding equipments or inducing cascading trippings, thus leaving some room for the application of corrective controls. Another implicit assumption is that the system will be stable in its post-contingency configuration so that there will be an opportunity to drive the system to a viable state through corrective controls. Since the system dynamics are not explicitly taken into account in the CSCOPF formulation, the latter is normally formulated conservatively, by imposing ad-hoc constraints on the magnitude of

Manuscript received May 02, 2008; revised August 30, 2008. First published February 13, 2009; current version published April 22, 2009. This paper presents research results of the Belgian Network DYSCO, funded by the Interuniversity Attraction Poles Programme, initiated by the Belgian State, Science Policy Office. The scientific responsibility rests with its authors. Paper no. TPWRS-00334-2008.

F. Capitanescu and L. Wehenkel are with the Department of Electrical Engineering and Computer Science (Montefiore Institute), University of Liège, Liège, Belgium (e-mail: capitane @montefiore.ulg.ac.be).

T. Van Cutsem is with the Department of Electrical Engineering and Computer Science (Montefiore Institute), University of Liège, Liège, Belgium, and also with the Fund for Scientific Research (FNRS).

Digital Object Identifier 10.1109/TPWRS.2008.2012181 post-contingency controls and/or the target feasible region. This may however lead to sub-optimalities and/or undetected risks of instability.

In this paper, we focus on long-term voltage security control, which has already received significant attention in the context of OPF [5]-[9] and to some extent in the context of security-constrained OPF [7] or dedicated methods [10], [11]. Most of these approaches consist in adding to the OPF problem some constraints ensuring a minimal voltage stability margin for the base case and/or for postulated contingencies. Optimal control actions to ensure voltage stability are then computed separately, either preventive ones [5]-[10] or corrective ones [5]-[7], [11]. However, most of these optimization approaches do not take into account system dynamics. There are some exceptions such as [10] and [11] where time-domain simulations are used to check voltage stability, but these references only consider preventive [10] or corrective controls [11], respectively, and not the combination of both. Methods incorporating the discretized system trajectory as constraints into the optimization itself have been proposed in [12] and [13]. Although general, this approach remains computationally demanding.

A first step towards efficient incorporation of system dynamics in the CSCOPF formulation was proposed in [14]. It consists in imposing constraints that ensure the existence and viability of the post-contingency short-term equilibrium of the system until corrective actions can start. Although necessary, these conditions are however not sufficient to obtain a stable transition towards a long-term equilibrium. Moreover, it may be difficult to determine beforehand how much the long-term viability constraints should be relaxed in the short term.

Determining optimal combinations of preventive and corrective controls while also ensuring system stability thus remains an open problem in the context of CSCOPF [15], [16]. In this paper, we propose an approach to determine an optimal combination of preventive and corrective control actions ensuring satisfactory dynamic transition of the system towards a feasible long-term equilibrium, if any of a set of postulated contingencies occurs. This approach combines static optimization with quasi steady-state simulation [17]. Information provided by the latter is used to iteratively modify the combination of preventive and corrective controls. This time-domain method has been used for its good trade-off between accuracy and speed but other (efficient) time-domain simulations could be used.

Furthermore, this paper focuses on generation rescheduling. Post-disturbance generation rescheduling can indeed be an effective countermeasure against long-term voltage instability provided that generators with some reserves are properly located (e.g., with respect to loads driving voltage instability). The proposed approach could however be extended to other countermeasures such as generator voltage control, shunt compensation switching or load shedding, by either adding them 
to the optimized controls or by taking them into account as automatic devices in the time-domain simulation.

Given the cost of generation rescheduling and the low probability of occurrence of contingencies, it is highly desirable to resort to post-disturbance control [4], [18]. However, an essential feature of these controls is their limited rate of change. In fact, the time needed to implement them may significantly decrease their ability to save a system from long-term voltage instability, for which speed of response is an issue [17]. As a result, more controls may be required and a main objective of this paper is to address this dynamic issue.

The paper is organized as follows. Section II deals with static optimization in preventive/corrective mode, while the incorporation of dynamic aspects is considered in Section III. Algorithms to obtain the sought combination of preventive and corrective controls are presented in Section IV. A detailed example is given in Section V, and Section VI concludes.

\section{Static Optimization OF PREVEnTIVE AND CORRECTIVE CONTROL ACTIONS}

\section{A. Preventive Controls Through CSCOPF}

The CSCOPF problem can be compactly stated as follows (see the Appendix for the detailed model used in our implementation):

$$
\begin{array}{ll} 
& \min f_{0}\left(\mathbf{x}_{0}, \mathbf{u}_{0}\right) \\
\text { s.t. } & \mathbf{g}_{k}\left(\mathbf{x}_{k}, \mathbf{u}_{k}\right)=\mathbf{0} \quad k=0, \ldots, c \\
& \mathbf{h}_{k}\left(\mathbf{x}_{k}, \mathbf{u}_{k}\right) \leq \mathbf{0} \quad k=0, \ldots, c \\
& \left|\mathbf{u}_{k}-\mathbf{u}_{0}\right| \leq T_{k}(d \mathbf{u} / d t)_{\max } \quad k=1, \ldots, c
\end{array}
$$

where $k=0$ corresponds to the pre-contingency configuration while $k=1, \ldots, c$ correspond to the $c$ post-contingency configurations, $f_{0}$ models the cost of preventive control actions, $\mathbf{x}_{k}$ is the vector of state variables (i.e., real and imaginary part of voltage at all buses), $\mathbf{u}_{0}$ (resp. $\mathbf{u}_{k}, k=1, \ldots, c$ ) is the vector of preventive (resp. corrective) control variables (e.g., generator active powers, generator terminal voltages, controllable transformer ratios, shunt reactances, etc.), $T_{k}$ is the assumed time horizon allowed for corrective control to ensure feasibility of the $k$ th post-contingency state and $(d \mathbf{u} / d t)_{\max }$ is the vector of maximum ramp rates of corrective controls.

Constraints (2) and (3) impose the feasibility (existence and viability) of the pre-contingency and corrected post-contingency states. Constraints (2) are mainly the ac power flow equations, while constraints (3) concern operation limits (e.g., limits on: control variables, branch currents, etc.). Inequalities (4) are "coupling" constraints between the preventive and corrective values of control variables aimed to prevent unrealistic variations of control variables in corrective mode. The product $T_{k}(d \mathbf{u} / d t)_{\max }$ is thus the vector of maximal allowed variations of control variables between the base case and $k$ th post-contingency state. Clearly, setting $T_{k}$ to zero is equivalent to resorting to pre-contingency controls only.

We assume the CSCOPF problem is solved by an iterative algorithm aimed to quickly identifying contingencies prone to be binding at the optimum and including those contingencies only into the optimization.

\section{B. Corrective Controls Through Post-Contingency OPF}

Assume that the system operates in a base case with the controls set to $\mathbf{u}_{0}$, solution of the optimization problem (1)-(3) limited to $k=0$, i.e., with the contingencies ignored. Assessing the effect of the contingencies may lead to one of the following three situations:

1) the operating constraints (2) and (3) are still satisfied after each contingency. There is no need for a preventive or corrective action. The system can be operated in the base case and no additional cost is incurred;

2) the constraints (2) and (3) are violated after some contingency $k>0$ but there exists at least one value of $\mathbf{u}_{k}$ satisfying (4) and allowing the constraints (2) and (3) to be met in the post-contingency post-correction situation. In other words, it is possible to meet the constraints by correcting the operating point after the contingency. Hence, no preventive action is needed and, again, there is no additional cost with respect to the base case;

3 ) the constraints (3) are violated after some contingency $k>$ 0 and there is no value of $\mathbf{u}_{k}$ satisfying (2), (3) and (4). Assuming that the CSCOPF problem is feasible, it is required to modify $\mathbf{u}_{0}$ and hence, there is a cost associated with security enhancement. The latter, however, is kept to a minimum by exploiting corrective controls to the greatest extent possible. As a result, some or all constraints (4) will be binding at the optimum.

Let us observe that in the second situation there is in general more than one value of $\mathbf{u}_{k}$ satisfying (2)-(4). In other words, the CSCOPF problem has multiple solutions, all equally optimal in terms of pre-contingency operating costs $f_{0}$.

To distinguish between the various solutions, one could add post-contingency control costs to the objective function. However, this would require to somehow account for the probability of occurrence of the contingency. Furthermore, it is widely agreed that in normal, pre-contingency conditions the main objective is to minimize operating costs while in the (comparatively short-lasting) emergency situations the main objective is to effectively eliminate constraint violations. Hence, we prefer to consider pre-contingency operating costs only and select the optimal post-contingency controls according to another criterion.

To this purpose, we first solve the CSCOPF formulation (1)-(4) so as to determine optimal settings for the preventive control variables $\mathbf{u}_{0}^{\star}$ while ensuring the existence of feasible corrective controls for each contingency. Then, in a second step, we freeze the preventive control settings to $\mathbf{u}_{0}^{\star}$, and compute for each contingency an optimal corrective control $\mathbf{u}_{k}^{\star}$. Note that this second step needs to be carried out only for those contingencies that would otherwise lead to constraint violations.

To determine appropriate corrective control actions, several objectives can be thought of: minimum post-contingency control deviations, minimum number of post-contingency control actions, minimum load shedding, etc. Since we focus on corrective generation rescheduling [4], [18], we propose to take as objective function the total deviation of generator active powers with respect to their pre-contingency values. This choice leads, 
for the $k$ th contingency, to the following post-contingency optimal power flow (PCOPF) problem:

$$
\begin{aligned}
\min _{\mathbf{x}_{k}, \mathbf{u}_{k}} & \mathbf{e}^{T} \\
\text { s.t. } & \mathbf{g}_{k}\left(\mathbf{u}_{k}, \mathbf{u}_{0} \mid=\mathbf{0}\right. \\
& \mathbf{h}_{k}\left(\mathbf{x}_{k}, \mathbf{u}_{k}\right) \leq \mathbf{0} \\
& \left|\mathbf{u}_{k}-\mathbf{u}_{0}\right| \leq \tau_{k}(d \mathbf{u} / d t)_{\max }
\end{aligned}
$$

where $\mathbf{u}_{0}$ is the vector of preventive control settings, $\mathbf{e}$ is a vector whose elements corresponding to generator active powers are equal to one and zero otherwise, and $\tau_{k}$ is the assumed time horizon allowed for corrective actions. Control variables, equality and inequality constraints (6)-(8) have the same meaning as in the CSCOPF formulation. The reason for having $\tau_{k}$ different from $T_{k}$ will appear in the sequel.

The objective (5) is a reasonable default choice, favoring an as small as possible corrective control effort. It makes sense in an emergency mode where speed of action is of concern, especially if system stability is threatened. Notice however, that alternative objective functions could be used together with the rate constraint (8). Changing this objective function leads to different post-contingency controls $\mathbf{u}_{k}^{\star}$ but does not affect the system operating costs $f_{0}$. Furthermore, if a contingency $k$ (belonging to the third situation previously described) constrains the CSCOPF optimum, there is no degree of freedom on $\mathbf{u}_{k}^{\star}$ (i.e., CSCOPF and PCOPF yield the same optimal corrective controls) and hence PCOPF objective function has no impact on pre-contingency operating costs.

Unless confusion is possible, we drop from the notation index $k$ referring to the considered contingency. We denote a solution of the PCOPF by $\left(\mathbf{x}^{\star}\left(\mathbf{u}_{0}, \tau\right), \mathbf{u}^{\star}\left(\mathbf{u}_{0}, \tau\right)\right)$, recalling that this solution depends on the parameters $\mathbf{u}_{0}$ and $\tau$.

Since we occasionally need to call the PCOPF under conditions where feasibility is not guaranteed, to quickly identify problem infeasibility and prevent numerical trouble we use a classical scheme combining relaxation [e.g., of constraints (7) and (8)] and penalization [4], not explicitly shown here.

\section{INCORPORATING DYNAMIC ASPECTS}

\section{A. Basic Assumptions}

In response to a disturbance happening at time $t_{0}$, we assume that:

- generator active powers can be changed after a delay $\Delta t$, assumed identical for all generators for simplicity;

- the rate of change of each production cannot exceed some value;

- the duration of the power adjustment cannot exceed some value $\tau$, also assumed identical for all generators.

Thus, generator active powers are assumed to vary linearly as sketched in Fig. 1, where three generations are adjusted from $P_{g i}^{0}$ to $P_{g i}(i=1, \ldots, 3)$ in at most $\tau$ seconds.

As we concentrate on long-term dynamics, we assume system short-term stability (possibly with the help of preventive and/or fast automatic post-contingency controls), so that no instability occurs before $t_{0}+\Delta t$, where generator active powers start being adjusted.

Furthermore, in order to determine whether the system responds in a stable way to the contingency and reaches in less

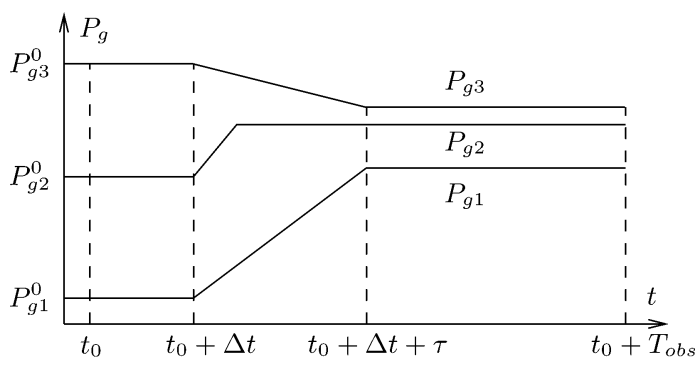

Fig. 1. Change in generation assumed in post-disturbance situation.

than $T_{\text {obs }}$ seconds (after the disturbance inception) a new longterm equilibrium satisfying specified operation limits, we simulate its dynamic response over the $\left[t_{0} t_{0}+T_{\text {obs }}\right]$ interval.

\section{B. Coupling Optimization and Dynamic Simulation: Principle}

The previously defined static optimization problems are coupled with dynamic simulation as follows.

Assume, for a given corrective action time $\tau$, that the postcontingency system evolution is voltage unstable while or after applying the computed optimal corrective actions. This means that the time left to counteract voltage instability was overestimated and stronger corrective controls have to be applied.

To make corrective actions more effective, we consider smaller values of $\tau$ in the PCOPF problem. In fact, reducing the value of $\tau$ in (8) has two effects. On one hand, tightening the bounds on control variations will lead to increasing the number of corrective controls and consequently resorting to less efficient ones. On the other hand, these more numerous controls will start acting at $t_{0}+\Delta t$. Clearly, the former effect is negative while the latter is positive. If the additional called up controls are much less efficient, no improvement is likely to result from a decrease of $\tau$. On the contrary, if the efficiency of the additional controls is not much lower, generation rescheduling will take place faster; hopefully, fast enough to avoid voltage instability [17]. As a simple example, a correction $\Delta P / 2$ applied simultaneously on two equivalent generators is more efficient than a correction $\Delta P$ on a single generator. Provision is made in the algorithms to detect situations where the negative effect would dominate.

We seek to determine an optimal combination of preventive and corrective controls such that, at the end of the observation time $T_{\mathrm{obs}}$, the system has reached a long-term equilibrium satisfying final operation limits. To this purpose, we build upon the following ideas:

- we assume that we are given a maximum time $T_{\max } \leq$ $T_{\text {obs }}-\Delta t$ for corrective controls to take place;

- we seek to determine the largest value of $T \in\left[0 \ldots T_{\max }\right]$ such that the post-disturbance evolution meets the above requirement. Indeed, the larger $T$, the smaller the preventive control cost;

- for a given value of $T$, we may compute preventive controls $\mathbf{u}_{0}^{\star}(T)$ by CSCOPF, use $\mathbf{u}_{0}^{\star}(T)$ with different values of $\left.\tau \in] 0 \ldots T_{\max }\right]$ to determine $\mathbf{u}^{\star}\left(\mathbf{u}_{0}^{\star}(T), \tau\right)$ by PCOPF, and check through dynamic simulation whether that control combination yields the desired response;

- among the values of $\tau$ yielding satisfactory system evolutions, we want to determine the largest one, so as to minimize post-contingency control. 


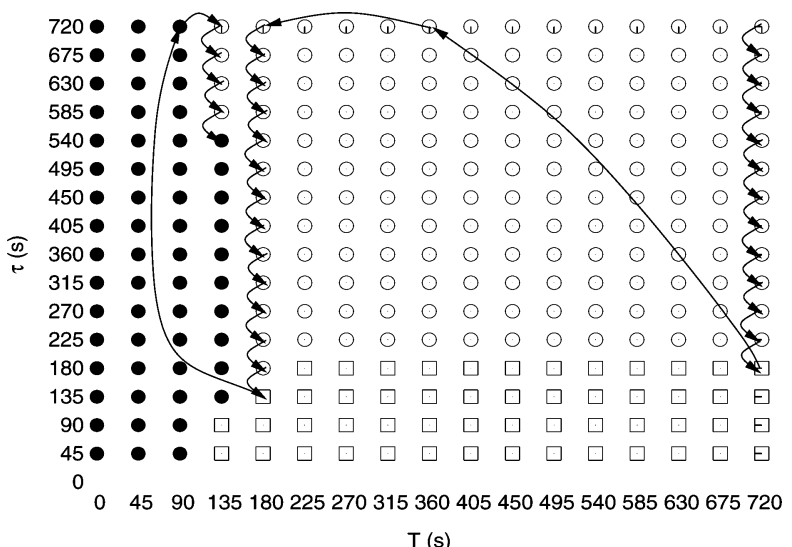

Fig. 2. Time horizon space $T-\tau$ relative to a contingency. $\bullet$ corresponds to stable system evolution towards a feasible equilibrium, o corresponds to voltage unstable system evolution, and $\square$ corresponds to infeasible PCOPF.

Algorithms implementing these ideas are given in Section IV.

Finally, operating rules may require the system to have in addition a minimum security margin with respect to instability. If a minimum security margin is required only for the base case the CSCOPF model (1)-(4) can be extended by adding the constraints (2) and (3) corresponding to the system operating at the minimum margin in pre-contingency state. Moreover, if the system has to ensure a minimum margin with respect to all $c$ contingencies, the "two-point" formulation of [7]-[9] can be used at the expense of duplicating (2) and (3) for each contingency.

\section{Algorithms to Determine Preventive AND CORRECTIVE CONTROLS}

Let us denote by $\Omega$ the subset of values of $(T, \tau) \in$ $\left.\left.\left[0 \ldots T_{\max }\right] \times\right] 0 \ldots T_{\max }\right]$ leading both to feasible CSCOPF and PCOPF, and by $\Omega_{s}$ its subset leading also to stable dynamics. Thus, we search for $T_{\max }^{s}=\max \left\{T: \exists(T, \tau) \in \Omega_{s}\right\}$ and $\tau_{\max }^{s}\left(T_{\max }^{s}\right)=\max \left\{\tau:\left(T_{\max }^{s}, \tau\right) \in \Omega_{s}\right\}$.

Given a combination $(T, \tau) \in \Omega$ and the corresponding $\mathbf{u}_{0}^{\star}(T)$ and $\mathbf{u}^{\star}\left(\mathbf{u}_{0}^{\star}(T), \tau\right)$ we check whether $(T, \tau)$ belongs to $\Omega_{s}$ as explained in Section III.

Note that if for a certain combination $(T, \tau)$ the system is declared unstable before $t_{0}+\Delta t$ we may conclude that for this $T$ there are no corrective control actions ensuring a stable behavior; in other words, we may conclude that $T_{\max }^{s}<T$. On the other hand, if $(T, \tau)$ leads to stable post-contingency dynamics, we may hope that larger values of $T$ and/or $\tau$ could also lead to stable behavior; hence we may conclude that $T_{\max }^{s} \geq T$ and $\tau_{\max }^{s}(T) \geq \tau$. Finally, if $(T, \tau)$ leads to an instability after $t_{0}+\Delta t$ it is hoped that smaller values of $\tau$ for the same $T$ will lead to a stable behavior.

\section{A. Illustration for a Single Contingency}

Fig. 2 illustrates the effect of $T$ and $\tau$ and the search of $\left(T_{\max }^{s}, \tau_{\max }^{s}\right)$ for a contingency analyzed in detail in Section V-C. Observe that PCOPF infeasibility cannot happen for $\tau \geq T$, while feasibility and even stable post-contingency dynamics are possible when $\tau<T$. Note that $\tau=0$ is not considered, since, if feasible, the PCOPF problem has the trivial solution $\mathbf{u}^{\star}=\mathbf{u}_{0}$ (no corrective action).
The point ( $T=135 \mathrm{~s}, \tau=540 \mathrm{~s}$ ) represents in this example the optimal combination of preventive and corrective controls. Arrows suggest how this point is determined iteratively, starting from the upper right corner of the diagram. It can be seen that for $T \in[180 \ldots 720]$ s no corrective actions are able to ensure post-contingency voltage stability, the system becoming voltage unstable too quickly after the start of corrective actions. On the other hand, for $T \leq 90 \mathrm{~s}$ the system is voltage stable without any corrective actions. For the optimal value $T=T_{\max }^{s}=135$ $\mathrm{s}$, the system behavior is stable for $\tau \in[135 \ldots 540] \mathrm{s}$, and (reasonably) diminishing $\tau$ stabilizes the system.

For the case shown in Fig. 2, no preventive action is taken for $T=360 \mathrm{~s}$ and $T=720 \mathrm{~s}$; hence, for the same $\tau$, the system behavior is the same for $T=360 \mathrm{~s}$ and $T=720 \mathrm{~s}$. Hence, to save computational time, the search of $\tau_{\max }^{s}$ is not performed for $T=360 \mathrm{~s}$.

\section{B. Algorithm for Handling a Single Contingency}

The goal of the algorithm, given in Fig. 3, is to search for a good approximation of $T_{\max }^{s}$ and $\tau_{\max }^{s}\left(T_{\max }^{s}\right)$ in the $\Omega$ subset.

The external loop looks for $T_{\max }^{s}$ by calling CSCOPF while the internal loop searches for $\tau_{\max }^{s}(T)$ by calling PCOPF. Inside both loops dynamic simulation is called to check the system time response. The external loop uses a bisection method (or binary search), based on the assumption that when a value of $T$ leads to instability for all values of $\tau$, then a larger value of $T$ will not help. On the contrary, because a similar assumption does not necessarily hold true for $\tau$, the internal search decreases $\tau$ by constant steps.

Note that to save computational time, the algorithm starts by checking, for each candidate value of $T$, whether the system becomes voltage unstable before generation rescheduling starts (i.e., before $t=t_{0}+\Delta t$ ). If this happens it can be concluded that for this value of $T$ no corrective controls are able to ensure voltage stability $\left.\forall \tau(T) \in] 0 \ldots T_{\max }\right]$.

The internal search for $\tau_{\max }^{s}(T)$ keeps on decreasing the value of $\tau$ until: 1) the system becomes voltage stable, 2) $\tau \leq 0$, or 3) the PCOPF becomes infeasible. This last condition is justified by the fact that if the PCOPF is infeasible for a given $\tau$, it will remain so also for smaller values.

Two particular outcomes of the algorithm are noteworthy:

1) no preventive control is needed to obtain a stable post-contingency evolution, which corresponds to $T_{\max }^{s}=T_{\max }$. It may even be possible that the contingency is not binding at the optimum in which case the CSCOPF optimum $\mathbf{u}_{0}^{\star}\left(T_{\max }\right)$ coincides with that of an OPF including base case constraints only;

2) preventive actions must be taken in order to enhance the base case such that the system withstands the contingency while considering corrective actions. This situation is revealed when, for $T=T_{\max }$, either the system becomes voltage unstable before the corrective actions can start or when corrective actions are unable to avoid voltage instability.

To simplify presentation some shortcuts to avoid useless computations are not detailed here, for instance when different values of $T$ (resp. $\tau$ ) lead to identical pre-contingency (resp. post-contingency) controls. Also, the internal search for $\tau_{\max }^{s}$ can be improved if the range of values leading to stable behavior can be assumed to lie in a connected interval. 


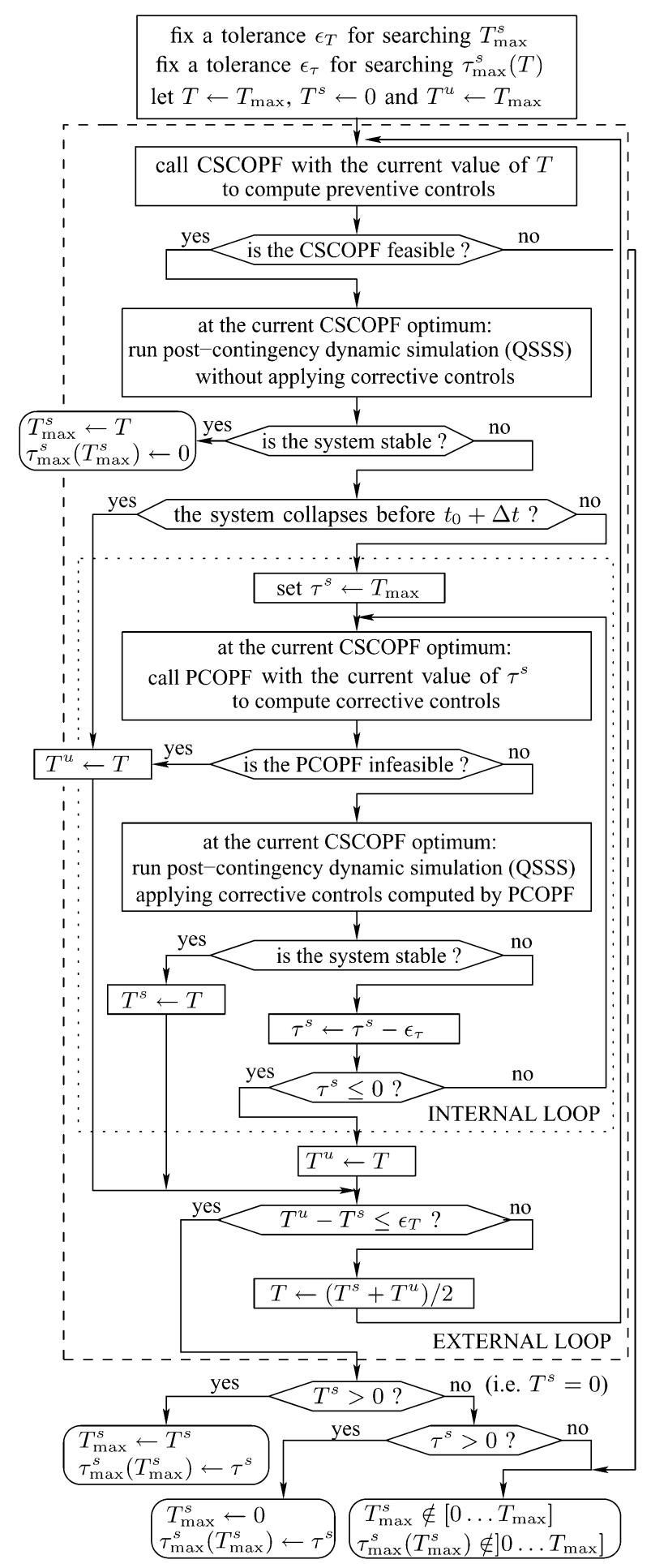

Fig. 3. Flowchart of the search algorithm of a valid optimal combination of preventive and corrective controls for a single contingency.

\section{Algorithm for Handling Several Contingencies}

The algorithm to determine an optimal combination of preventive and corrective actions for CSCOPF problem (1)-(4) is provided in Fig. 4 (notation as in Section IV-B). Observe that the algorithm of Fig. 3 computing an optimal combination of preventive and corrective actions for a given contingency is embedded in the algorithm of Fig. 4.

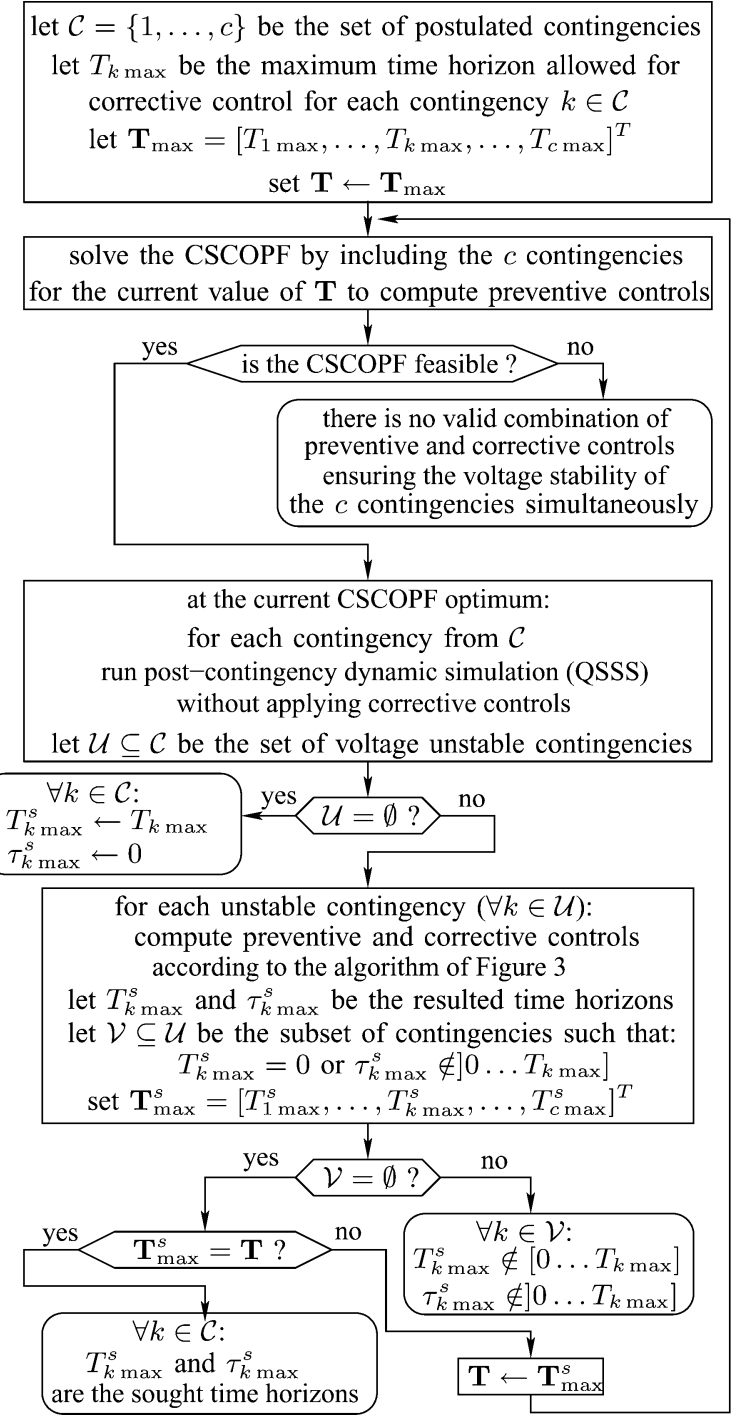

Fig. 4. Flowchart of the search algorithm of a valid optimal combination of preventive and corrective controls for a list of contingencies.

This algorithm terminates whenever one of following conditions is met: 1) for the current vector $\mathbf{T}$ of time horizons, for which CSCOPF has been solved, all $c$ contingencies can be taken care of by corrective actions, 2) for at least one contingency, $T_{k \max }^{s}=0$ and no corrective action yields a post-contingency voltage stable evolution, or 3 ) the CSCOPF becomes infeasible indicating that no combination of preventive and corrective controls is able to ensure voltage stability with respect to all $c$ contingencies simultaneously.

\section{NUMERICAL RESULTS}

\section{A. Modelling, Objectives, and Criteria}

The proposed approach is illustrated through tests on the Nordic32 system [19], whose one-line diagram is shown in Fig. 5. In this system voltage instability is caused by a large power transfer in the corridor linking the North+Equiv and Central+South regions (see Fig. 5). Hence, a preventive or corrective increase of generation in the Central or Southern areas, with a corresponding adjustment of Northern generators, 


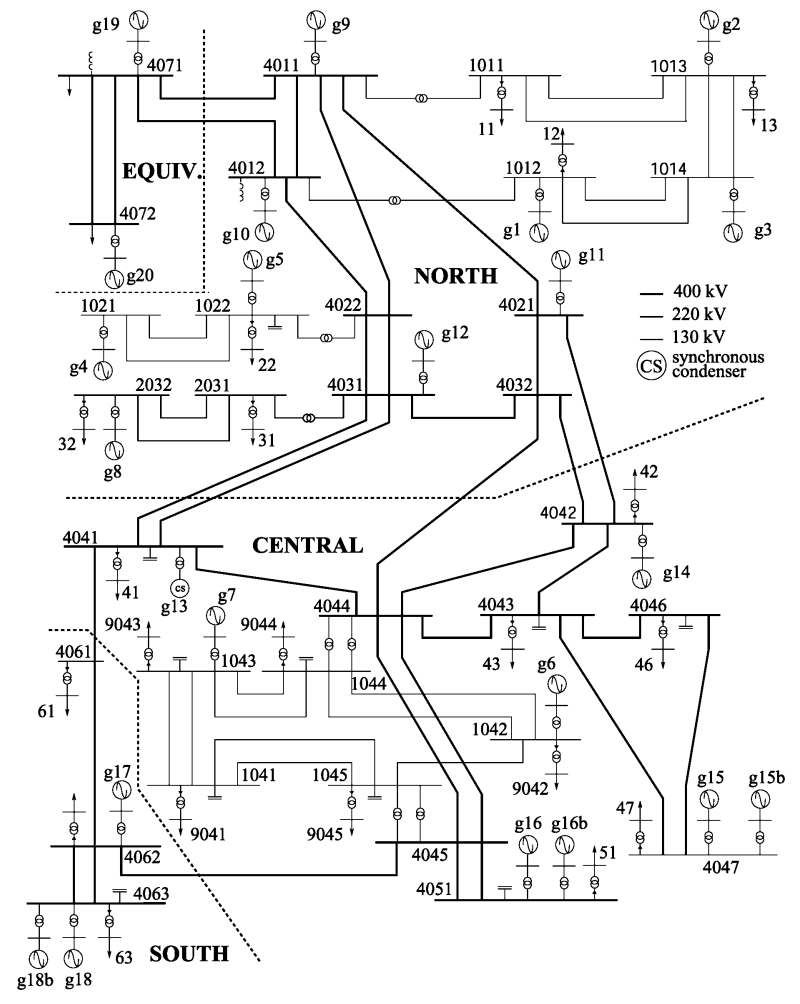

Fig. 5. One-line diagram of Nordic32 system.

improves voltage stability. In corrective mode, however, this action must be fast enough with respect to load power restoration and generator excitation reduction [17]. As a result of stressed operating conditions, the $\mathrm{N}-1$ incidents considered hereafter are equivalent to $\mathrm{N}-2$ or more severe incidents in a real-life system.

The model includes 55 buses, 23 generators, 22 loads, 52 lines, 31 transformers (four with controllable ratio), and 11 shunts, respectively.

The objective of the CSCOPF problem is the overall generation cost (each generator has a quadratic cost). The control variables are the generator active powers. Equality constraints are the bus active/reactive power balance equations. Inequality constraints stem from bounds on generator active/reactive powers, voltage magnitudes, and branch currents. We use the same branch thermal limits in all states, while bus voltage magnitudes are allowed to vary between 0.95 p.u. (resp. 0.92 p.u.) and 1.10 p.u. in pre-contingency (resp. post-contingency) state, except at buses 1044 and 1045, which are controlled by load tap changers (LTCs) and hence vary in tighter intervals in all states.

The above assumptions for CSCOPF are also made in PCOPF which focuses on minimum post-contingency generation rescheduling (together with post-contingency feasibility).

Both OPF problems are solved using the interior-point method described in [20].

The long-term system response to both contingency and corrective generation rescheduling has been obtained by quasi steady-state simulation (QSSS) [17]. The long-term dynamics are driven by LTCs and overexcitation limiters (OELs), both acting with various delays. In CSCOPF and PCOPF, loads are represented as constant power at the transmission level, while in QSSS an exponential model is considered for the 22 loads, represented behind their distribution transformers, all equipped with LTCs. Constant reactive power limits have been considered for generators in CSCOPF and PCOPF, but their values are updated from the more detailed QSSS model. Participation of generators to frequency control is taken into account in QSSS, CSCOPF, and PCOPF. QSSS extends over $T_{\mathrm{obs}}=\Delta t+T_{\max }=900 \mathrm{~s}$ and uses a time step of $1 \mathrm{~s}$. Voltage instability is declared if some transmission voltages reach a low value of 0.75 p.u., or short-term equilibrium is lost [17].

As regards corrective generation rescheduling, the following assumptions were made:

1) each generator is able to reschedule linearly, up and down, $50 \%$ of its nominal power over one hour;

2) productions start being changed (simultaneously) $\Delta t=$ $180 \mathrm{~s}$ after the contingency occurrence at $t_{0}=10 \mathrm{~s}$;

3 ) in both CSCOPF and PCOPF, the time horizon of corrective actions varies between 0 (i.e., no corrective action, except automatic controls) and $T_{\max }=720 \mathrm{~s}$;

4) tolerances for searching $T_{\max }^{s}$ and $\tau_{\max }^{s}\left(T_{\max }^{s}\right)$ are taken as $\epsilon_{T}=\epsilon_{\tau}=45 \mathrm{~s}$.

\section{B. Case 1: Outage of Line 4022-4031}

We start by illustrating step-by-step the proposed procedure in the case of a single contingency, namely the loss of line 4022-4031 (see Fig. 5).

First, CSCOPF is solved with constraints for that contingency and with the time horizon of corrective actions $T$ set to its maximum value $T_{\max }=720 \mathrm{~s}$. After setting the system to the so obtained initial operating point, the contingency is simulated without corrective action. Voltage collapse takes place at $t=380 \mathrm{~s}$.

Next, PCOPF is used to compute the minimum corrective controls ensuring feasibility with the specified time horizon $\tau=$ $T_{\max }=720 \mathrm{~s}$. The system response to both the disturbance and the so determined generation rescheduling, applied from $t=190 \mathrm{~s}$ on, is determined using QSSS. It is found that the system loses stability at $t=696 \mathrm{~s}$, i.e., $316 \mathrm{~s}$ later than without corrective controls. This happens while generation rescheduling is still taking place, which means that the pace of the latter should be quickened.

According to the algorithm of Fig. 3, $\tau$ is decreased by steps of $\epsilon_{\tau}$, new corrective actions are determined by PCOPF for each value of $\tau$, and QSSS is performed with the so obtained rescheduling. This sequence is repeated until the system evolution is stabilized. The optimal corrective actions eventually able to do so correspond to $\tau=540 \mathrm{~s}$. At this point, it is found that the contingency is not binding, since the CSCOPF objective keeps the same value as in the base case. Hence, no preventive action is required $\left(T_{\max }^{s}=720 \mathrm{~s}\right.$ ); corrective controls suffice to stabilize the system.

Table I provides, for successive time horizons $\tau$, the generation rescheduling at PCOPF optimum, together with the QSSS outcome. Note that while $\tau$ is decreased from 720 to $585 \mathrm{~s}$, the power corrections of all generators do not change since none of them is reaching its (decreasing) upper limit. Clearly, to save computing time, QSSS is called only when corrective controls change. Note also that in this example the total generation rescheduling does not increase when $\tau$ is decreased from 585 to $540 \mathrm{~s}$, indicating that generators g16 and g16b are equally 
TABLE I

CASE 1: CoRrective Generation Changes (IN MW) GIVEN BY PCOPF FOR $T=720 \mathrm{~s}$

\begin{tabular}{|c|c|c|}
\hline generator & \multicolumn{3}{|c|}{ time horizon $\tau(\mathrm{s})$} \\
& $720 \ldots 585$ & 540 \\
\hline \hline $\mathrm{g} 7$ & 9.3 & 9.3 \\
\hline $\mathrm{g} 16$ & & 4.0 \\
\hline g16b & 56.5 & 52.5 \\
\hline \hline total & 65.8 & 65.8 \\
\hline \hline QSSS diagnosis & unstable at $t=696 \mathrm{~s}$ & stable \\
\hline
\end{tabular}

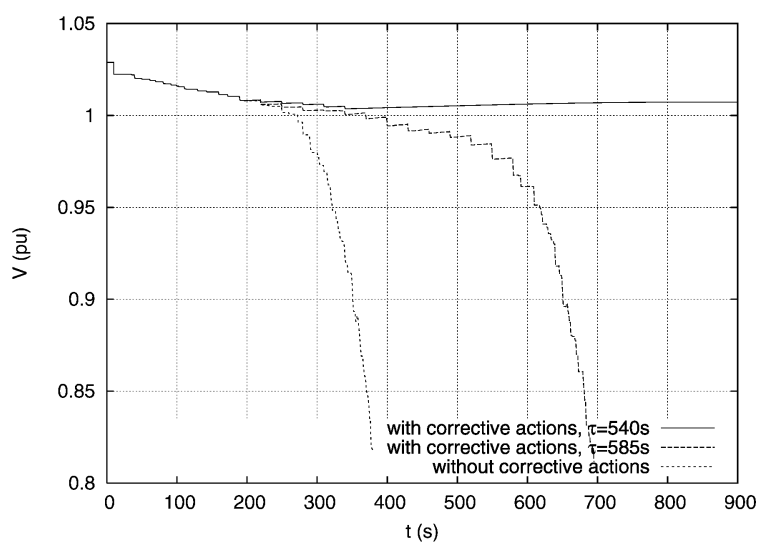

Fig. 6. Case 1: voltage evolution at bus 4047 for various corrective actions.

effective. This results from the fact that they are located very close to each other (see Fig. 5).

Fig. 6 shows the voltage evolution at bus 4047, experiencing the largest drop, in three cases: unstable without corrective actions, marginally unstable with corrective actions corresponding to $\tau=585 \mathrm{~s}$ and marginally stable with corrective actions corresponding to $\tau=540 \mathrm{~s}$. In the latter case, corrective controls were fast enough to counteract the effect of LTCs and OELs. Obviously, this cannot be found from PCOPF alone. The latter provides post-contingency operating points with all voltages in the requested range $([0.92 \ldots 1.10] \mathrm{pu})$ but the so obtained longterm equilibria are not all attracting. The results clearly illustrate the benefit of coupling optimization with time-domain simulation.

\section{Case 2: Outage of Line 4041-4044}

For this contingency, the successive scenarios checked in the course of searching a valid optimal combination of preventive and corrective actions were shown in Fig. 2.

Table II provides the generation change at the PCOPF optimum, together with the collapse time, for various values of $\tau$, when $T$ is set to $T_{\max }=720 \mathrm{~s}$. Note that all these corrective schemes fail to save (or even extend significantly the survival of) the system, since generation rescheduling starts at $t=190$ $\mathrm{s}$ while the system collapses at $t=215 \mathrm{~s}$ in the absence of corrective actions. However, from the PCOPF perspective, the results confirm that the smaller the amount of power available for rescheduling, the higher the number of rescheduled generators and the higher the total generation shift.

As seen from Fig. 2, the iterations stopped for $\tau=180 \mathrm{~s}$ owing to the divergence of the PCOPF algorithm, indicating that the available corrective actions are insufficient to ensure
TABLE II

CASE 2: Corrective Generation Changes (IN MW) GIVEN BY PCOPF FOR $T=720 \mathrm{~s}$

\begin{tabular}{|c|c|c|c|c|c|}
\hline generator & \multicolumn{5}{|c|}{ time horizon $\tau(\mathrm{s})$} \\
& 720 & 450 & 360 & 270 & 225 \\
\hline \hline $\mathrm{g} 1$ & -47.9 & -50.0 & -40.0 & -30.0 & -25.0 \\
\hline $\mathrm{g} 2$ & & -15.2 & -30.0 & -22.5 & -18.7 \\
\hline $\mathrm{g} 3$ & & & & -26.2 & -21.9 \\
\hline $\mathrm{g} 6$ & & 21.1 & 20.0 & 15.0 & 12.5 \\
\hline $\mathrm{g} 7$ & 9.4 & 9.4 & 9.4 & 7.5 & 6.2 \\
\hline $\mathrm{g} 9$ & & & & -2.4 & -41.0 \\
\hline $\mathrm{g} 10$ & & -13.7 & -21.8 & -30.0 & -25.0 \\
\hline $\mathrm{g} 14$ & & & & 26.2 & 21.9 \\
\hline $\mathrm{g} 15$ & & 4.9 & 19.5 & 22.5 & 18.7 \\
\hline $\mathrm{g} 15 \mathrm{~b}$ & & 4.9 & 19.5 & 22.5 & 18.7 \\
\hline $\mathrm{g} 16$ & 43.1 & 43.7 & 35.0 & 26.2 & 21.9 \\
\hline $\mathrm{g} 16 \mathrm{~b}$ & 70.0 & 43.7 & 35.0 & 26.2 & 21.9 \\
\hline $\mathrm{g} 17$ & & & & 10.7 & 18.7 \\
\hline $\mathrm{g} 18$ & & & & & 17.7 \\
\hline $\mathrm{g} 18 \mathrm{~b}$ & & & & & 17.7 \\
\hline $\mathrm{g} 19$ & -25.0 & -0.6 & -3.0 & -9.4 & -7.8 \\
\hline \hline total & 195.4 & 207.5 & 233.2 & 277.3 & 315.3 \\
\hline \hline unstable at $t=$ & $217 \mathrm{~s}$ & $219 \mathrm{~s}$ & $219 \mathrm{~s}$ & $218 \mathrm{~s}$ & $217 \mathrm{~s}$ \\
\hline
\end{tabular}

post-contingency feasibility. Therefore, the pre-contingency operating point must be modified by specifying in CSCOPF a time horizon $T$ smaller than $T_{\max }=720 \mathrm{~s}$.

The preventive actions determined by CSCOPF for the successive values of $T$ determined by the bisection search are shown in Table III, together with the diagnosis from QSSS. In all cases, no corrective action was considered. For $T=720$ $\mathrm{s}$ and $T=360 \mathrm{~s}$, no preventive action is taken, the CSCOPF optimum being the same as in the pre-contingency situation. The next point checked by the bisection method is $T=180$ $\mathrm{s}$, as shown in Fig. 2. For this value of $T$ the pre-contingency rescheduling is still insufficient: the collapse time is increased by $18 \mathrm{~s}$ only, and no corrective rescheduling is able to restore stability. On the other hand, for $T=90 \mathrm{~s}$, the system is stable without corrective actions. Note that the large number of control changes could be reduced, at the detriment of operation cost, by re-running CSCOPF with the small changes inhibited.

The last value checked by bisection is $T=135 \mathrm{~s}$, where combined pre- and post-contingency reschedulings succeed restoring voltage stability. The corresponding generation changes determined by PCOPF are given in Table IV for various values of $\tau$, with $T$ set to $135 \mathrm{~s}$. The system is marginally stable for $\tau=540 \mathrm{~s}$. Again, these results demonstrate the benefit of rescheduling more generators at the same time.

From Tables III and IV it results that the optimal trade-off of preventive and corrective controls corresponds to $T_{\max }^{s}=135$ $\mathrm{s}$ and $\tau_{\max }^{s}=540 \mathrm{~s}$, respectively.

The time evolution of the voltage at bus 4046, experiencing the largest post-contingency drop, is shown in Fig. 7, for two operating points stemming from CSCOPF and corresponding to $T=T_{\max }=720 \mathrm{~s}$ and $T=135 \mathrm{~s}$, respectively. In both cases the curves with and without corrective actions are provided. For $T=720 \mathrm{~s}$, they are almost indiscernible due to the little time left for corrective actions.

\section{Case 3: Outage of Line 4011-4021}

This line outage is more severe in the sense that without rescheduling the system collapses only $41 \mathrm{~s}$ after the disturbance is applied. This does not leave time to correctively 
TABLE III

Case 2: Preventive Generation Changes (IN MW) GIVEN BY CSCOPF (NO CORRECTIVE ACTION)

\begin{tabular}{|c|c|c|c|c|}
\hline generator & \multirow{4}{|c|}{ time horizon $T$ (s) } \\
& 720 and 360 & 180 & 90 & 135 \\
\hline \hline $\mathrm{g} 1$ & & -2.8 & -11.2 & -6.9 \\
\hline $\mathrm{g} 2$ & & -1.4 & -5.6 & -3.5 \\
\hline $\mathrm{g} 3$ & & -2.5 & -10.3 & -6.3 \\
\hline $\mathrm{g} 4$ & & -1.8 & -7.4 & -4.5 \\
\hline $\mathrm{g} 5$ & & -0.8 & -3.3 & -2.1 \\
\hline $\mathrm{g} 6$ & & 3.5 & 14.0 & 8.6 \\
\hline $\mathrm{g} 7$ & & 2.6 & 6.8 & 5.6 \\
\hline $\mathrm{g} 8$ & & -3.2 & -11.9 & -7.7 \\
\hline $\mathrm{g} 9$ & & -2.4 & -9.8 & -6.0 \\
\hline $\mathrm{g} 10$ & & -2.8 & -11.2 & -6.9 \\
\hline $\mathrm{g} 11$ & & -0.8 & -3.0 & -1.9 \\
\hline $\mathrm{g} 12$ & & -0.9 & -3.3 & -2.1 \\
\hline $\mathrm{g} 14$ & & 1.9 & 8.3 & 4.9 \\
\hline $\mathrm{g} 15$ & & 2.0 & 8.7 & 5.2 \\
\hline $\mathrm{g} 15 \mathrm{~b}$ & & 2.0 & 8.7 & 5.2 \\
\hline $\mathrm{g} 16$ & & 7.0 & 28.2 & 17.4 \\
\hline $\mathrm{g} 16 \mathrm{~b}$ & & 1.2 & 4.9 & 3.0 \\
\hline $\mathrm{g} 17$ & & 0.4 & 2.6 & 1.3 \\
\hline $\mathrm{g} 18$ & & 0.3 & 2.0 & 1.0 \\
\hline $\mathrm{g} 18 \mathrm{~b}$ & & 0.3 & 2.0 & 1.0 \\
\hline $\mathrm{g} 19$ & & -1.2 & -5.0 & -3.1 \\
\hline $\mathrm{g} 20$ & & -7.8 & -31.8 & -19.5 \\
\hline \hline total & & 49.9 & 200.3 & 123.7 \\
\hline \hline QSSS & unstable & unstable & stable & unstable \\
diagnosis & at $t=215 \mathrm{~s}$ & at $t=233 \mathrm{~s}$ & & at $t=360 \mathrm{~s}$ \\
\hline & & & \multicolumn{2}{c|}{} \\
\hline
\end{tabular}

TABLE IV

Case 2: Corrective Generation Changes (IN MW) GIVEN BY PCOPF FOR $T=135 \mathrm{~s}$

\begin{tabular}{|c|c|c|c|c|c|}
\hline generator & \multicolumn{5}{|c|}{ time horizon $\tau(\mathrm{s})$} \\
& 720 & 675 & 630 & 585 & 540 \\
\hline \hline $\mathrm{g} 7$ & 3.8 & 3.8 & 3.8 & 3.8 & 3.8 \\
\hline $\mathrm{g} 16$ & 0.5 & 4.9 & 9.4 & 13.8 & 18.2 \\
\hline $\mathrm{g} 16 \mathrm{~b}$ & 70.0 & 65.6 & 61.2 & 56.9 & 52.5 \\
\hline $\mathrm{g} 20$ & -6.6 & -6.7 & -6.8 & -6.9 & -7.0 \\
\hline \hline total & 80.9 & 81.0 & 81.2 & 81.4 & 81.5 \\
\hline \hline QSSS & unstable at & unstable at & unstable at & unstable at & stable \\
diagnosis & $t=399 \mathrm{~s}$ & $t=448 \mathrm{~s}$ & $t=510 \mathrm{~s}$ & $t=523 \mathrm{~s}$ & \\
\hline
\end{tabular}

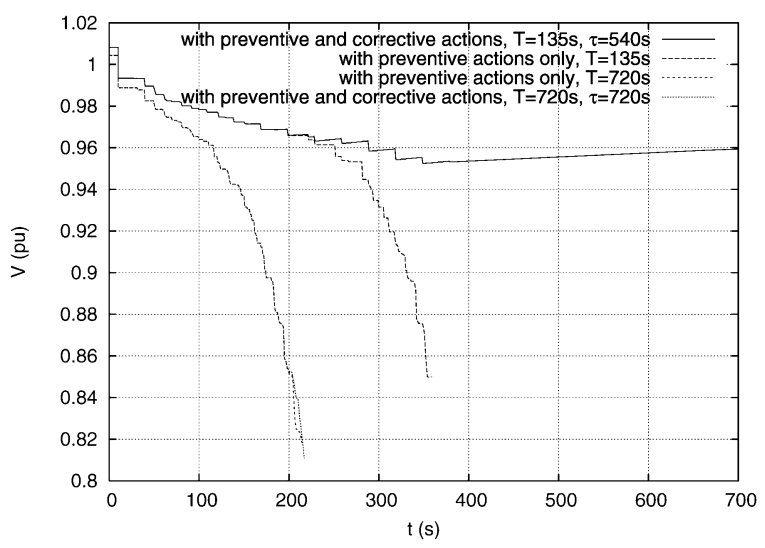

Fig. 7. Case 2: voltage evolution at bus 4046 for four combinations of preventive and corrective actions.

reschedule generators and hence some preventive actions are needed to secure the system.

The preventive generation changes determined by CSCOPF are given in Table $\mathrm{V}$, for the successive values of $T$ chosen by
TABLE V

Case 3: Preventive Generation Changes (IN MW) GIVEN BY CSCOPF (No CORRECTIVE ACTION)

\begin{tabular}{|c|c|c|c|c|c|}
\hline generator & \multicolumn{5}{|c|}{ time horizon $T(\mathrm{~s})$} \\
& 720 and 360 & 180 & 90 & 45 & 0 \\
\hline \hline g1 & & -14.2 & -26.1 & -32.0 & -39.3 \\
\hline g2 & & -7.1 & -13.1 & -16.0 & -19.8 \\
\hline g3 & & -13.0 & -23.9 & -29.3 & -36.1 \\
\hline g4 & & -9.5 & -17.3 & -21.2 & -27.9 \\
\hline g5 & & -3.7 & -6.7 & -8.1 & -9.9 \\
\hline g6 & & 7.5 & 13.8 & 17.0 & 21.1 \\
\hline g7 & & 4.3 & 6.8 & 8.1 & 9.3 \\
\hline g8 & & -9.2 & -13.7 & -15.1 & -20.5 \\
\hline g9 & & -12.6 & -23.2 & -28.5 & -34.9 \\
\hline g10 & & -14.2 & -26.1 & -32.0 & -39.3 \\
\hline g11 & & 7.7 & 13.7 & 16.7 & 21.9 \\
\hline g12 & & 2.2 & 4.2 & 5.3 & 7.2 \\
\hline g14 & & 13.5 & 24.7 & 30.6 & 35.3 \\
\hline g15 & & 11.7 & 21.5 & 25.6 & 29.4 \\
\hline g15b & & 11.7 & 21.5 & 25.6 & 29.4 \\
\hline g16 & & 13.7 & 25.3 & 31.4 & 42.9 \\
\hline g16b & & 2.3 & 4.3 & 5.3 & 7.3 \\
\hline g17 & & 9.3 & 17.2 & 21.4 & 29.4 \\
\hline g18 & & 8.8 & 16.3 & 20.2 & 27.8 \\
\hline g18b & & 8.8 & 16.3 & 20.2 & 27.8 \\
\hline g19 & & -6.4 & -11.8 & -14.5 & -17.7 \\
\hline g20 & & -40.5 & -74.3 & -91.2 & -111.4 \\
\hline \hline total & & 232.3 & 421.6 & 515.5 & 645.5 \\
\hline \hline QSSS & unstable at & unstable at & unstable at & stable & stable \\
diagnosis & $t=51 \mathrm{~s}$ & $t=107 \mathrm{~s}$ & $t=172 \mathrm{~s}$ & & \\
\hline & & & & & \\
\hline
\end{tabular}

the bisection method and in the absence of any corrective action. For comparison purposes, the last column shows the values provided by CSCOPF when setting $T=0$, i.e., when relying on preventive actions only. As expected, this leads to a higher rescheduling. The optimal value is $T_{\max }^{s}=45 \mathrm{~s}$ and as a matter of fact it does not require corrective actions, the post-contingency system evolution being voltage stable with preventive controls only.

Fig. 8 shows the evolution of the voltage at bus 4022 , for the five combinations in Table V. As expected, with the preventive controls corresponding to $T=0$, the voltage settles at a higher value than with the controls corresponding to $T=45 \mathrm{~s}$. It can be also seen that, the smaller the horizon $T$, the stronger the preventive actions and the later the collapse time. Finally, note that for $T>90$ s the system collapses before corrective actions can be launched.

\section{E. Case 4: Stabilizing Several N-1 Contingencies}

In this last example, the algorithm of Fig. 4 is used to control a set of $37 \mathrm{~N}-1$ contingencies.

First, CSCOPF is solved with all contingencies included and $T$ set to $T_{\max }=720 \mathrm{~s}$ for each of them. At the so obtained CSCOPF solution, each contingency is simulated with QSSS without corrective action. Seven contingencies are found to cause voltage instability. They are listed in Table VI with the collapse time shown in the column labeled "iteration 1".

Next, optimal corrective actions are determined for each of the seven contingencies by the algorithm of Fig. 3. This computation can be skipped for the first three contingencies since the system collapses before any corrective action can be launched. It is found to also fail for the next two contingencies due to insufficient time left to corrective actions to save the system. The last two can be stabilized. 


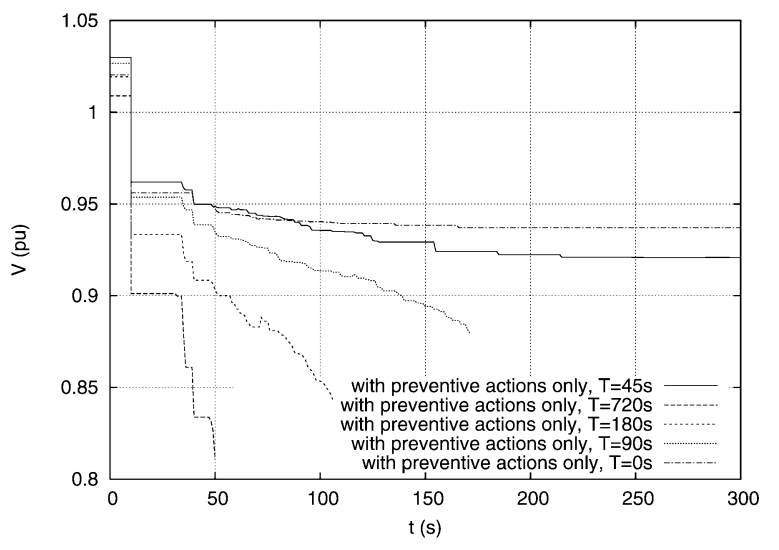

Fig. 8. Case 3: voltage evolution at bus 4022 for various preventive actions.

TABLE VI

CASE 4: RESUlTS OF CSCOPF AND QSSS

\begin{tabular}{|c|c|c|c|c|}
\hline \multirow{2}{*}{$\begin{array}{c}\text { loss of } \\
\text { line }\end{array}$} & $\begin{array}{c}T \\
(\mathrm{~s})\end{array}$ & $\begin{array}{c}\text { QSSS } \\
\text { diagnosis }\end{array}$ & $\begin{array}{c}T_{\max }^{\text {max }}(\mathrm{s}) \\
\text { iteration 2 }\end{array}$ & $\begin{array}{c}\text { QSSS } \\
\text { diagnosis }\end{array}$ \\
\hline \hline $4011-4021$ & 720 & unstable $(51 \mathrm{~s})$ & 45 & stable \\
\hline $4021-4042$ & 720 & unstable $(142 \mathrm{~s})$ & 45 & unstable $(512 \mathrm{~s})$ \\
\hline $4032-4044$ & 720 & unstable $(151 \mathrm{~s})$ & 45 & unstable $(281 \mathrm{~s})$ \\
\hline $4031-4041$ & 720 & unstable $(208 \mathrm{~s})$ & 135 & stable \\
\hline $4041-4044$ & 720 & unstable $(215 \mathrm{~s})$ & 135 & stable \\
\hline $4022-4031$ & 720 & unstable $(380 \mathrm{~s})$ & 720 & stable \\
\hline $4031-4032$ & 720 & unstable $(397 \mathrm{~s})$ & 720 & stable \\
\hline
\end{tabular}

Thus, the first five contingencies require preventive changes in system operating point. To this purpose, for each contingency, the time horizon $T_{\max }^{s}$ is searched using the algorithm of Fig. 3. The results are given in the column of Table VI labeled "iteration 2". For the last two contingencies, $T_{\max }^{s}=720 \mathrm{~s}$ since there exist corrective actions saving the system (the same holds true for the remaining 30 contingencies which are stable even without corrective actions).

Finally, CSCOPF is solved again using the individually computed time horizons $T_{\max }^{s}$. A new optimal operating point is obtained as a trade-off between two contingencies: the outage of lines 4021-4042 and 4032-4044, respectively. Table VII shows the preventive actions needed to stabilize each of the two contingencies separately, and both simultaneously. The results show an expected compromise of preventive actions to handle both contingencies; the main differences between the three production schemes appear on generators $\mathrm{g} 8, \mathrm{~g} 11, \mathrm{~g} 12, \mathrm{~g} 14, \mathrm{~g} 15, \mathrm{~g} 15 \mathrm{~b}$, g16, and g20.

\section{CONCLUSION}

Static preventive-corrective optimizations cannot account for the speed at which post-contingency corrective actions are applied. It may happen that the post-contingency system evolution is voltage unstable because the statically computed corrective controls are not acting fast enough even when applied at the maximum allowed rate.

To handle such situations in the case of long-term voltage stability, an approach coupling static optimization with time-domain simulation has been proposed. By adjusting the time horizons in CSCOPF and PCOPF, it yields a valid optimal combination of preventive and corrective actions such that, if any of the postulated contingencies occurs, by applying CSCOPF-
TABLE VII

CASE 4: GENERATION CHANGES (IN MW) GIVEN By CSCOPF

\begin{tabular}{|c|c|c|c|}
\hline generator & \multicolumn{3}{|c|}{ dealing with contingencies } \\
& $4032-4044$ & $4021-4042$ & BOTH \\
\hline \hline $\mathrm{g} 1$ & -27.6 & -32.0 & -30.9 \\
\hline $\mathrm{g} 2$ & -13.9 & -16.0 & -15.5 \\
\hline $\mathrm{g} 3$ & -25.4 & -29.3 & -28.4 \\
\hline $\mathrm{g} 4$ & -19.2 & -21.2 & -20.9 \\
\hline $\mathrm{g} 5$ & -8.0 & -8.1 & -8.3 \\
\hline $\mathrm{g} 6$ & 18.6 & 17.0 & 18.6 \\
\hline $\mathrm{g} 7$ & 8.1 & 8.1 & 8.1 \\
\hline $\mathrm{g} 8$ & -26.3 & -15.1 & -21.2 \\
\hline $\mathrm{g} 9$ & -24.1 & -28.5 & -27.3 \\
\hline $\mathrm{g} 10$ & -27.5 & -32.0 & -30.8 \\
\hline $\mathrm{g} 11$ & -6.9 & 16.7 & 4.4 \\
\hline $\mathrm{g} 12$ & -8.3 & 5.3 & -1.8 \\
\hline $\mathrm{g} 14$ & 18.0 & 30.6 & 24.6 \\
\hline $\mathrm{g} 15$ & 19.0 & 25.6 & 22.9 \\
\hline $\mathrm{g} 15 \mathrm{~b}$ & 19.0 & 25.6 & 22.9 \\
\hline $\mathrm{g} 16$ & 59.3 & 31.4 & 49.0 \\
\hline $\mathrm{g} 16 \mathrm{~b}$ & 10.7 & 5.3 & 8.4 \\
\hline $\mathrm{g} 17$ & 23.1 & 21.4 & 23.0 \\
\hline $\mathrm{g} 18$ & 21.0 & 20.2 & 21.3 \\
\hline $\mathrm{g} 18 \mathrm{~b}$ & 21.0 & 20.2 & 21.3 \\
\hline $\mathrm{g} 19$ & -12.3 & -14.5 & -13.9 \\
\hline $\mathrm{g} 20$ & -77.8 & -91.2 & -87.6 \\
\hline \hline total & 495.2 & 515.5 & 511.2 \\
\hline & & & \\
\hline
\end{tabular}

based preventive actions and PCOPF-based corrective actions, the system will be able to reach the feasible state determined by optimization (which may include limits on voltage magnitudes and branch currents as well).

The proposed formulation has been successfully tested on cases where generation rescheduling is used to counteract voltage instability. The so determined corrective actions should be implemented through an automatic system protection scheme, avoiding the delays introduced by operator interventions.

The approach is computationally intensive (especially if detailed time-domain simulation is used) but it could be significantly speeded up by distributing the coupled PCOPF-QSSS sub-problems relative to different contingencies over different processors [4], [21].

\section{APPENDIX}

Let $n, g, t$, and $s$ be the number of: buses, generators, transformers with controllable ratio, and shunts, respectively. We formulate the CSCOPF problem (1)-(4) with voltages expressed in rectangular coordinates

$$
\underline{V}_{i}=e_{i}+j f_{i}, \quad i=1, \ldots, n .
$$

We use generator active powers, generator terminal voltages, controllable transformer ratios, and shunt reactances as control variables.

The objective function (1) is the minimum generation cost

$$
\min \sum_{i=1}^{g}\left(c_{0 i}+c_{1 i} P_{G i}^{0}+c_{2 i}\left(P_{G i}^{0}\right)^{2}\right) .
$$

Constraints (2) encompass the active and reactive power flow equations, written for each bus $i(i=1, \ldots, n)$ and each system configuration $k(k=0, \ldots, c)$

$$
P_{G i}^{k}-P_{L i}-\left[\left(e_{i}^{k}\right)^{2}+\left(f_{i}^{k}\right)^{2}\right] \sum_{j \in N_{i}^{k}}\left(G_{s i j}+G_{i j}\right)
$$




$$
\begin{array}{r}
+\sum_{j \in N_{i}^{k}}\left[\left(e_{i}^{k} e_{j}^{k}+f_{i}^{k} f_{j}^{k}\right) G_{i j}+\left(f_{i}^{k} e_{j}^{k}-e_{i}^{k} f_{j}^{k}\right) B_{i j}\right]=0 \\
Q_{G i}^{k}-Q_{L i}+\left[\left(e_{i}^{k}\right)^{2}+\left(f_{i}^{k}\right)^{2}\right]\left[B_{s i}+\sum_{j \in N_{i}^{k}}\left(B_{s i j}+B_{i j}\right)\right] \\
-\sum_{j \in N_{i}^{k}}\left[\left(e_{i}^{k} e_{j}^{k}+f_{i}^{k} f_{j}^{k}\right) B_{i j}+\left(e_{i}^{k} f_{j}^{k}-f_{i}^{k} e_{j}^{k}\right) G_{i j}\right]=0
\end{array}
$$

where $N_{i}^{k}$ is the set of neighbor buses of bus $i$ in state $k$, other notations being self-explanatory.

Constraints (3) include operational limits on branch currents and voltage magnitudes

$$
\begin{gathered}
\left(G_{i j}^{2}+B_{i j}^{2}\right)\left[\left(e_{i}^{k}-e_{j}^{k}\right)^{2}+\left(f_{i}^{k}-f_{j}^{k}\right)^{2}\right] \leq\left(I_{i j}^{\max }\right)^{2} \\
\left(V_{i}^{\min }\right)^{2} \leq\left(e_{i}^{k}\right)^{2}+\left(f_{i}^{k}\right)^{2} \leq\left(V_{i}^{\max }\right)^{2} \\
i=1, \ldots, n, \quad j \in N_{i}^{k}, \quad k=0, \ldots, c
\end{gathered}
$$

and physical limits of power system devices

$$
\begin{array}{rlrl}
P_{G i}^{\min } \leq P_{G i}^{k} \leq P_{G i}^{\max }, & & i=1, \ldots, g & k=0, \ldots, c \\
Q_{G i}^{\min } \leq Q_{G i}^{k} \leq Q_{G i}^{\max }, & & i=1, \ldots, g \quad k=0, \ldots, c \\
r_{i}^{\min } \leq r_{i}^{k} \leq r_{i}^{\max }, & & i=1, \ldots, t \quad k=0, \ldots, c \\
b_{i}^{\min } \leq b_{i}^{k} \leq b_{i}^{\max }, & & i=1, \ldots, s \quad k=0, \ldots, c .
\end{array}
$$

Although not shown explicitly, the variable $r_{i}$ intervenes in the CSCOPF formulation through the terms $G_{s i j}, B_{s i j}, G_{i j}$ and $B_{i j}$, while $b_{i}$ intervenes through the term $B_{s i}$ only.

Finally, the coupling constraints (4) take on the form

$$
\left|P_{G i}^{k}-P_{G i}^{0}\right| \leq T_{k}\left(d P_{G i}^{k} / d t\right)_{\max } i=1, \ldots, g \quad k=1, \ldots, c .
$$

\section{REFERENCES}

[1] J. Carpentier, "Contribution à l'étude du dispatching économique," Bull. Societé Française d'Electricité, vol. 3, pp. 431-447, 1962.

[2] H. W. Dommel and W. F. Tinney, "Optimal power flow solutions," IEEE Trans. Power App. Syst., vol. PAS-87, no. 10, pp. 1866-1876, 1968.

[3] O. Alsac and B. Stott, "Optimal load flow with steady-state security," IEEE Trans. Power App. Syst., vol. PAS-93, no. 3, pp. 745-751, 1974.

[4] A. J. Monticelli, M. V. P. Pereira, and S. Granville, "Security-constrained optimal power flow with post-contingency corrective rescheduling," IEEE Trans. Power Syst., vol. 2, no. 1, pp. 175-182, Feb. 1987

[5] X. Wang, G. C. Ejebe, J. Tong, and J. G. Waight, "Preventive/corrective control for voltage stability using direct interior point method," IEEE Trans. Power Syst., vol. 13, no. 3, pp. 878-883, Aug. 1998.

[6] Z. Feng, V. Ajjarapu, and D. J. Maratukulam, "A comprehensive approach for preventive and corrective control to mitigate voltage collapse," IEEE Trans. Power Syst., vol. 15, no. 2, pp. 791-797, May 2000.

[7] E. Vaahedi, Y. Mansour, C. Fuchs, S. Granville, M. de Lujan Latore, and H. Hamadanizadeh, "Dynamic security constrained optimal power flow/VAR planning," IEEE Trans. Power Syst., vol. 16, no. 1, pp. 38-43, Feb. 2001.
[8] W. D. Rosehart, C. A. Canizares, and V. H. Quintana, "Multiobjective optimal power flows to evaluate voltage security costs in power networks," IEEE Trans. Power Syst., vol. 18, no. 2, pp. 578-587, May 2003.

[9] F. Milano, C. A. Canizares, and M. Invernizzi, "Multiobjective optimization for pricing system security in electricity markets," IEEE Trans. Power Syst., vol. 18, no. 2, pp. 596-604, May 2003.

[10] F. Capitanescu and T. Van Cutsem, "Preventive control of voltage security margins: A multi-contingency sensitivity-based approach," IEEE Trans. Power Syst., vol. 17, no. 2, pp. 358-364, May 2002.

[11] C. Moors and T. Van Cutsem, "Determination of optimal load shedding against voltage instability," in Proc. 13th PSCC Conf., Trondheim, Norway, 1999, pp. 993-1000.

[12] E. De Tuglie, M. La Scala, and P. Scarpellini, "Real-time preventive actions for the enhancement of voltage-degraded trajectories," IEEE Trans. Power Syst., vol. 14, no. 2, pp. 561-568, May 1999.

[13] E. De Tuglie, M. Dicorato, M. La Scala, and P. Scarpellini, “A corrective control for angle and voltage stability enhancement on the transient time-scale," IEEE Trans. Power Syst., vol. 15, no. 4, pp. 1345-1353, Nov. 2000.

[14] F. Capitanescu and L. Wehenkel, "Improving the statement of the corrective security-constrained optimal power flow problem," IEEE Trans. Power Syst., vol. 22, no. 2, pp. 887-889, May 2007.

[15] E. Vaahedi and H. M. Zein El-Din, "Considerations in applying optimal power flow to power system operation," IEEE Trans. Power Syst., vol. 4, no. 2, pp. 694-703, May 1989.

[16] J. A. Momoh, R. J. Koessler, M. S. Bond, B. Stott, D. Sun, A. Papalexopoulos, and P. Ristanovic, "Challenges to optimal power flow," IEEE Trans. Power Syst., vol. 12, no. 1, pp. 444-455, Feb. 1997.

[17] T. Van Cutsem and C. Vournas, Voltage stability of Electric Power Systems. Boston, MA: Kluwer, 1998.

[18] S. G. Johansson, J. E. Daalder, D. Popovic, and D. J. Hill, "Avoiding voltage collapse by fast active power rescheduling," Int. J. Elect. Power Energy Syst., vol. 19, no. 8, pp. 501-509, 1997.

[19] Long-Term Dynamics, Phase II, 1995, CIGRE Task Force 38.02.08.

[20] F. Capitanescu, M. Glavic, D. Ernst, and L. Wehenkel, "Interior-point based algorithms for the solution of optimal power flow problems," Elect. Power Syst. Res., vol. 77, no. 5-6, pp. 508-517, Apr. 2007.

[21] M. Rodrigues, O. R. Saavedra, and A. Monticelli, "Asynchronous programming model for the concurrent solution of the security constrained optimal power flow problem," IEEE Trans. Power Syst., vol. 9, no. 4, pp. 2021-2027, Nov. 1994.

Florin Capitanescu received the electrical power engineering degree from the University "Politehnica" of Bucharest, Bucharest, Romania, in 1997 and the $\mathrm{Ph} . \mathrm{D}$. degree from the University of Liège, Liège, Belgium, in 2003.

His main research interests include optimization methods and voltage stability.

Thierry Van Cutsem (M'94-SM'03-F'05) received the electrical-mechanical engineering and Ph.D. degrees from the University of Liège, Liège,, Belgium.

He is now an Adjunct Professor at the University of Liège. Since 1980, he has been with the Fund for Scientific Research (FNRS), of which he is now a Research Director. His research interests are in power system dynamics, stability, security, optimization, and real-time control, in particular voltage stability and security.

Louis Wehenkel (M'93) received the electrical engineering (electronics) degree in 1986 and the Ph.D. degree in 1990, both from the University of Liège, Liège, Belgium.

He is a full Professor of electrical engineering and computer science at the University of Liège. His research interests lie in the fields of stochastic methods for systems and modeling, machine learning, and data mining, with applications in power systems planning, operation and control, and bioinformatics. 